Stamatiadis, N., Sturgill, R., Amiridis, K., and Taylor, T. (2017). "Estimating Constructability Review Benefits for Highway Projects." In: LC3 2017: Volume I - Proceedings of the Joint Conference on Computing in Construction (JC3), July 4-7, 2017, Heraklion, Greece, pp. 347-355. DOI: https://doi.org/10.24928/JC3-2017/0007.

\title{
ESTIMATING CONSTRUCTABILITY REVIEW BENEFITS FOR HIGHWAY PROJECTS
}

\author{
Nikiforos Stamatiadis ${ }^{1}$, Roy Sturgill ${ }^{2}$, Kiriakos Amiridis $^{3}$, and Timothy Taylor ${ }^{4}$
}

\begin{abstract}
Constructability review is a process used in the design phase of a project in order to interject construction knowledge and address potential issues prior to construction. This typically occurs with a team or panel of constructability reviewers. Current staffing and budgetary constraints have resulted in state transportation agencies being very careful about disturbing the existing project development process with practices that seemingly lie outside the main process, such as constructability review. An issue that constructability reviews face is the lack of any documented savings. Over the past decade, the Kentucky Transportation Cabinet has streamlined the constructability review process by relying on a smaller team or single reviewer per project and recently taken on an effort to estimate monetary benefits from such reviews. This paper discusses the evaluation of constructability reviews at the project level by comparing change order percentages on projects reviewed versus those not being reviewed. This approach showed a clear indication that there are monetary savings associated with constructability reviews resulting in a conservative savings estimate of 1.25 percent of the project cost without including the additional inherent savings in time, lessons learned or other aspects not readily quantifiable. This evidence presents that a streamlined constructability review process and team can still provide savings to a project. A regression model was also developed in this work to estimate the potential monetary gains from the constructability review comments but additional analysis is needed to improve accuracy. There is potential that this model could be used to further streamline the process by identifying and focusing on projects where constructability review savings could be maximized.
\end{abstract}

Keywords: Constructability review, design, quality assurance.

\section{INTRODUCTION}

Highway projects are planned, designed and constructed through a phased project development process aiming to deliver the most appropriate project for the setting. Part of this process relies not only on the expertise of the design team members but on the timeliness with which expertise is sought during the various stages of the project development process. Past work has documented the significant cost and time benefits of integrating construction expertise throughout the project development phases and especially in the design phases of a project (Gambatese et al. 2007). In an effort to

1 Professor, Department of Civil Engineering, University of Kentucky, Lexington, KY USA, nick.stamatiadis@uky.edu

2 Research Engineer, Kentucky Transportation Center, University of Kentucky, Lexington, KY USA, roy.sturgill@uky.edu

3 Graduate Research Assistant, Department of Civil Engineering, University of Kentucky, Lexington, KY USA, kiriakos.amiridis@uky.edu

4 Associate Professor, Department of Civil Engineering, University of Kentucky, Lexington, KY USA, tim.taylor@uky.edu 
capture such benefits, the Kentucky Transportation Cabinet (KYTC) has initiated a constructability review process where design documents are reviewed before the project reaches construction to identify potentially constructability issues. These reviews target both constructability issues and value engineering concepts and are conducted through small teams or individual reviewers. However, the existing process is more of an ad hoc approach that lacks a systematic means for collecting the required data and identifying potential benefits. Moreover, KYTC has available at most four reviewers so they cannot review all projects. KYTC recently developed a Constructability Review Database that allows for a systematic cataloguing of results from constructability reviews, analysis of their findings with rating and cost associations, and an ability to develop lessons learned from prior experiences (Stamatiadis et al. 2013).

The recent economic issues that several transportation agencies face due to the diminished roadway funds and increased need for road improvements require a critical examination of the project development process. To address this issue, several State Departments of Transportation (DOTs) have undertaken a variety of efforts and processes, including constructability reviews. The main goal of a constructability review is to critically examine and evaluate design options and then detect areas where benefits can be materialized. Project oversights and minimization of problems during construction are two major areas of focus several DOTs have focused (Anderson and Fisher 1997). Agencies have employed a systematic review of projects at various phases of the project development process that concentrate on identifying potential disputes that could lead to scope changes resulting in construction issues. Dunston et al. (2002) also demonstrated that the benefit/cost ratio of constructability reviews is greater than two. The same study also showed that effective constructability reviews have the potential to decrease project duration and improve the quality of the constructed facility. Despite the possible benefits of such reviews, NCHRP Report 390 found that only 23 percent of state DOTs use a formal Constructability Review process (Anderson and Fisher 1997).

The relationship between the ability to influence design options and associated cost is well documented in construction literature. The earlier issues are identified, the easier and less costly it will be to address them. It is therefore imperative to plan such reviews as early as possible to maximize their potential benefits. Projects approaching the construction phase become less flexible in changing various elements and thus the ability to impact cost and delivery time is diminished. Even though reviews conducted after the final design and before construction may still identify oversights, the changes at that time would likely result in additional costs and time for the project to be completed. Proper timing of constructability reviews is imperative to maximize benefits and avoid future issues.

This study addresses the benefits from constructability reviews through a comparison of projects with and without reviews as well as a review of case studies aiming to quantify these benefits. Disadvantages of conducting a constructability reviews, such as additional time for development, were disregarded because the constructability review process occurs outside of the main project development functions, i.e., it is not allowed to delay the project, and constructability comments are assessed and incorporated as seen applicable by project managers and thus, revisions are not required. 


\section{METHODOLOGY}

\subsection{Case Study Selection}

A comprehensive set of example projects for case analysis was identified through consultation with the study oversight panel. Projects were selected to include a variety of construction and design aspects as well scope and budget. The criteria for case selection include the project characteristics, the reviewer, the stage of the project, the number of review comments and the project location.

The project characteristics focused on identifying cases with a variety of elements in order to provide for a varied data set. Typical issues that were considered during the selection were project type, density of surrounding development, estimated construction cost, project designer, highway district, project manager, and project origination.

The stage of the project was also considered during the selection process to ensure that the case studies were selected among projects that have undergone a constructability review in the past few years. The selection of these projects was considered appropriate, since the final design would likely have been completed and the project possibly begun construction and thus allowing for an analysis of possible change orders and cost items. This provided for an accurate estimation of the impact that each review comment had on the cost of the project, and identify any potential shortcomings of the reviews completed.

Several criteria were used to select the cases starting with the number of review comments per project. The assumption is few comments resulting from a constructability review are likely a product of a design with high quality and little room for value added from the review. The threshold of ten comments was used for case study selection, since the analysis of the database indicated an average number of nine comments per review (118 reviews with a total of 1,110 comments). Two additional considerations were also included in the selection process. First, reviews were selected from each of the KYTC reviewers to get a cross-section of reviews from individuals with slightly varied backgrounds. The final criterion used to select cases was to get a geographical crosssection with the reviews to address any location specific issues.

The selected cases are shown in Table 1. A full description of the case study selection process is provided in Stamatiadis et al. (2013)

Table 1. Selected cases for analysis.

\begin{tabular}{|c|c|c|c|c|}
\hline $\begin{array}{l}\text { Case } \\
\text { No. }\end{array}$ & Phase of Review & $\begin{array}{l}\text { No. of } \\
\text { Comments }\end{array}$ & Project Type & Construction Est. \\
\hline 1 & Final Joint Inspection & 18 & Major Widening & $\$ 19,510,000.00$ \\
\hline 2 & Check Print & 36 & Major Widening & $\$ 41,250,000.00$ \\
\hline 3 & Final Joint Inspection & 11 & Safety & $\$ 675,000.00$ \\
\hline 4 & Final Joint Inspection & 3 & New Route & $\$ 12,120,000.00$ \\
\hline 5 & Check Print & 13 & New Route & $\$ 12,120,000.00$ \\
\hline 6 & Final Joint Inspection & 15 & Bridge Replacement & $\$ 850,000.00$ \\
\hline 7 & Final Joint Inspection & 13 & Bridge Replacement & $\$ 400,000.00$ \\
\hline 8 & Preliminary Line and Grade & 12 & Relocation & $\$ 45,450,000.00$ \\
\hline 9 & Final Joint Inspection & 20 & Bridge Replacement & $\$ 900,000.00$ \\
\hline
\end{tabular}




\subsection{Benefit Metrics}

To properly evaluate the nine cases and quantify their impact, a set of benefit metrics were established. The analysis focused on the valuation of the constructability review program both at the project and comment level.

The project level analysis concentrated on a comparative analysis between projects that were reviewed through the constructability review program and those that were not reviewed. Data for projects from 2007 to 2012 were compared to projects that were reviewed through the constructability program from 2010 through 2012 . The comparison was made by investigating the percentage of cost increase (or decrease) from the as-bid project cost to the final cost inclusive of change order adjustments. Any difference between these two categories of projects (reviewed versus not reviewed) would demonstrate a relationship between the constructability review program and any savings that could be noted.

The comment level analysis examined each comment and assessed both its quantitative and qualitative impact. The quantitative valuation was based on identifying the issues and costs associated with the comment if it was not addressed until the project was already under construction. In other words, if the problem, concern, or question were to occur during construction how would it have been addressed. From this analysis, the value of the comment could be determined by calculating the algebraic difference between the costs of addressing the comment during design versus addressing it during construction. Essentially, this analysis involved addressing what-if scenarios and developing change order type cost estimates to evaluate savings. The second, more general benefit metric was qualitative valuations of the comments categorized into three distinct groups (Table 2). These groups are defined by the level of corrective actions required during construction for not addressing the comments in design. The corrective actions might entail additional project communication, additional project documentation, additional project costs, change orders, additional project time, and project disputes or claims. These values were determined through feedback of personnel experienced in KYTC construction practices.

Table 2. Qualitative value level description.

\begin{tabular}{|c|c|}
\hline $\begin{array}{l}\text { Qualitative } \\
\text { Level }\end{array}$ & Description of Corrective Actions \\
\hline Low & $\begin{array}{l}\text { Corrective action may require additional project communication or clarification, } \\
\text { but can be completed without a change order. Project management staff efforts } \\
\text { would be minimal to rectify the situation. }\end{array}$ \\
\hline Medium & $\begin{array}{l}\text { Corrective action may incur minor project cost or time increases by change order } \\
\text { but the overall effects are considered average. The average change order results in } \\
\text { a } 3.5 \text { percent increase to the project. Project management staff would incur } \\
\text { additional documentation and time to rectify the situation. }\end{array}$ \\
\hline High & $\begin{array}{l}\text { Corrective action will result in large additions to the project in cost and/or time, } \\
\text { and would have potential for leading to project disputes or claims. Project } \\
\text { management staff would incur excessive amounts of added documentation and time } \\
\text { to rectify the situation. May result in additional tension between the contractor and } \\
\text { project management staff. }\end{array}$ \\
\hline
\end{tabular}




\section{RESULTS}

\subsection{Project Level}

The project level analysis compared projects that were reviewed against similar ones that were not reviewed. The analysis examined the change orders submitted for each project and estimated their impact as a percent of the total project cost. While change orders may not capture all changes or problems occurring on a project, the majority of those impacting the project cost would be represented.

Projects from 2007 through 2012 were identified and reviewed. The collected data included as-bid project cost and cost modifications by change orders. There was also information available to determine whether the project was complete and what design item series (an indication of project type and development process) was for the project. The available constructability review database allowed comparing these datasets in multiple ways and across multiple variables such as reviewer, district, completion status, or item number series. The amount of change orders as a percentage of the as-bid project cost was calculated and reported in Table 3Table in various categories of concern. The Item \# is a KYTC mechanism for tracking types of projects and their associated steps though the project development process. Projects with numbers less than 3000 and greater than 7000 will typically go through the entire design process and potentially are reviewed, while all others are traditionally maintenance projects that may not require constructability reviews. 
Table 3. Change orders as percent of project budget for project level evaluation.

\begin{tabular}{|c|c|c|c|c|}
\hline \multicolumn{5}{|c|}{ All Projects } \\
\hline \multirow[b]{2}{*}{ Reviewed } & \multicolumn{4}{|l|}{ Project Series } \\
\hline & All Projects & Item\#<3000 & Item\# $>7000$ & Item $\#>3000,<7000$ \\
\hline Yes & 3.383 & 3.794 & 1.902 & No Reviews \\
\hline No & 4.403 & 4.490 & 5.932 & 5.309 \\
\hline \multicolumn{5}{|c|}{ Completed Projects } \\
\hline \multirow[b]{2}{*}{ Reviewed } & \multicolumn{4}{|l|}{ Project Series } \\
\hline & All Projects & Item\#<3000 & Item $\#>7000$ & Item $\#>3000,<7000$ \\
\hline Yes & 3.012 & 3.546 & 0.074 & No Reviews \\
\hline No & 4.427 & 4.781 & 6.647 & 4.181 \\
\hline
\end{tabular}

Based on the data in Table 3, projects reviewed through the constructability review program incur a lower amount of change orders (on average 1.25 percent) than projects that were not reviewed. This percentage cannot directly be referred to as savings for projects that were reviewed because it is likely that changes were made based on the constructability review comments adding work or items during design that would have otherwise been added during construction by change order. The KYTC change order procedures indicate that change order items are acceptable at 110 percent of the average unit bid prices. An estimate of the value of the constructability review program for 20102012 can be derived utilizing the assumption that the reviews saved this 10 percent premium on the 1.25 percent in change order additions (Table 4). This estimation is extremely conservative. In addition, much of the value in constructability review is not accounted for here such as construction management time savings, designer lessons learned, and schedule delays saved.

Table 4. Estimated savings of the constructability review program by letting year.

\begin{tabular}{llll}
\hline Letting Year & $\begin{array}{l}\text { Bid Amount for Projects } \\
\text { Reviewed }\end{array}$ & $\begin{array}{l}1.25 \% \text { Estimated Price } \\
\text { Reduction }\end{array}$ & $\begin{array}{l}\text { Savings } \\
(10 \% \text { Premium })\end{array}$ \\
\hline 2010 & $\$ 112,060,060.98$ & $\$ 1,400,750.76$ & $\$ 140,075.08$ \\
2011 & $\$ 232,134,684.84$ & $\$ 2,901,683.56$ & $\$ 290,168.36$ \\
2012 & $\$ 88,625,270.91$ & $\$ 1,107,815.89$ & $\$ 110,781.59$ \\
\hline
\end{tabular}

\subsection{Comment Level}

The comment level analysis can provide for a more discrete analysis of the reviews even though this approach requires several assumptions. Each comment was reviewed and through a scenario-based analysis a savings estimate was developed. Comments were first evaluated quantitatively and then qualitatively. From the 141 comments analyzed, 73 were evaluated quantitatively because they were the only ones with available quantitative data. Various approaches were utilized to determine the value of each comment with an underlying objective to determine the impact the problem, issue, or ambiguity would have during construction.

Two approaches were utilized for estimating the value of comments related to omitted work or bid items. The most straightforward approach was when the bid item was not included. In this case, the KYTC average unit bid prices (AUBP) were used to estimate what costs would have been added to the project at a 10 percent premium, i.e. using a 110 percent of the AUBP. The benefit accrued from the comment was only the 10 percent premium savings that would occur due to correction prior to construction. The second approach involved comments for which omitted work simply meant additional quantity for a bid item already included in the project. Unless the omission 
affected the current bid quantity by more than 25 percent, by specification, no price adjustment is warranted during construction. In these cases where existing quantities were not changed by more than 25 percent no benefit was accrued for the comment. It can easily be inferred that economies of scale would apply to a quantity increase and therefore the comment does entail a direct benefit to the project; however, it is not quantifiable in this case.

The final approach taken to determine the value of a comment was for those that involved the simple elimination of bid items. The benefit in these cases simply entailed the quantities eliminated multiplied by the corresponding AUBP rates. If any items also had to be added after the comment eliminations were made, these were added at the normal AUBP rate according to the same reasoning above.

The quantities and values of these 73 comments were computed using the appropriate approach among those noted above. The data indicates that most of the comments resulted in a benefit of less than $\$ 2,000$ (52 comments of the 73 or 71.2 percent) with only eight comments with benefits over $\$ 10,000$ (11 percent). However, these 52 comments below $\$ 2,000$ only account for 4.2 percent of the quantified savings while the eight comments over $\$ 10,000$ account for 85.3 percent of the calculated savings. Having a majority of the dataset account for the smallest portion of the value determined, makes the values over $\$ 10,000$ appear as outliers to the data. However, this is most likely due to the small number of cases and comments analyzed. This data variability also affects the regression analysis discussed below.

As previously mentioned, all comments reviewed were assigned a qualitative value according to Table 2. A cross-examination of the qualitative scores by the estimated value indicates that most comments with low values are also those with a low qualitative level. There are few comments with high qualitative level and large benefit value (4 percent). Additional comparison and cross-examinations of the value with the comment types and comment categories did not produce any significant trends.

\subsection{Probabilistic Analysis}

An effort was undertaken to develop a prediction model of quantifiable benefits in order to estimate the value and benefits of the constructability reviews. The analysis presented here is based on the 73 quantified comments. The values obtained for the 73 comments range from $\$ 12$ to $\$ 166,000$. The majority of these values are below $\$ 2,000$ (71.2 percent). The significance level for the statistical analyses described next is assumed to be at the 95-percent level, i.e., p-values must be lower than 0.05 in order to be considered statistically significant. The statistical analysis was conducted with the SPSS software. The purpose of this effort is to develop a predictive model utilizing both categorical and quantitative variables as predictors and regression analysis is considered the most appropriate approach in this case, since there are no other suitable processes that could be used to address both categorical and quantitative variables.

Several attempts were made to develop a regression model in order to predict the possible constructability review benefits utilizing the available variables. With the goal of finding a parsimonious model, the Forward Selection approach was used to add predictors into the model according to their individual $\mathrm{t}$-statistic and analyzing their inclusion effects on the Goodness of Fit. Table 5 shows the progression of the models used in this effort and the equation that is considered the best fit for the existing data (Model 4). The data set allowed for six potential explanatory variables, which are: Error Type, Category Type, Severity Type, Quality Level, Number of Comments, and Construction Estimate. The first four variables are categorical and the other two, 
quantitative. In regression analysis, categorical variables are addressed through the use of dummy variables and coding, since their values are indicative more of a presence than a rank order.

Table 5. Models for statistical analysis.

\begin{tabular}{|c|c|c|c|c|}
\hline Model & \multicolumn{3}{|c|}{ Equation } & $R^{2}$ \\
\hline \multirow{3}{*}{1} & \multicolumn{3}{|c|}{ Quant Value $=93,486.667+b \times$ Quality_Level } & \multirow[t]{3}{*}{0.481} \\
\hline & Low & Medium & High & \\
\hline & $-92,331.775$ & $-71,928.761$ & 0 & \\
\hline \multirow{3}{*}{2} & \multicolumn{3}{|c|}{$\begin{aligned} \text { Quant Value }= & 88,612.353+b \times \text { QualityLevel } \\
& +3.392 \times 10^{-12} \\
& \times e^{\text {Number of Comments }}\end{aligned}$} & \multirow{3}{*}{0.541} \\
\hline & Low & Medium & High & \\
\hline & $-91,956.808$ & $-72,741.147$ & 0 & \\
\hline \multirow[t]{3}{*}{3} & $\begin{aligned} \text { Quant Value }= & 87,94 \\
& +0.0 \\
& \times(N \\
& \times C o 1\end{aligned}$ & $\begin{array}{l}.907+b \times \text { Quali } \\
67818494 \\
\text { mber of Comm } \\
\text { str Est })^{2}\end{array}$ & Level & \multirow[t]{3}{*}{0.542} \\
\hline & Low & Medium & High & \\
\hline & $-91,613.982$ & $-72,304.816$ & 0 & \\
\hline \multirow{6}{*}{4} & Quant Value $=$ & $\begin{array}{l}08,101.091+b \times \\
-c \times e^{\text {Number of }} \\
-d \times \text { Category } 1\end{array}$ & $\begin{array}{l}\text { ualityLevel } \\
\text { nments } \\
\text { pe }\end{array}$ & \multirow{6}{*}{0.602} \\
\hline & Low & Medium & High & \\
\hline & $-103,112.229$ & $-77,172.621$ & 0 & \\
\hline & $\mathrm{c}$ & $2.971 \times 10^{-1}$ & & \\
\hline & $\begin{array}{l}\text { Design \& } \\
\text { Construction }\end{array}$ & $\begin{array}{l}\text { Drainage \& } \\
\text { Pavement }\end{array}$ & Other & \\
\hline & $-12,653.322$ & $-18,884.604$ & 0 & \\
\hline
\end{tabular}

The statistical analysis conducted here provides some indication that there is the potential for developing prediction models for estimating the benefit of the reviewed comments based on various attributes of the comment. The limited data used here allows for reasonably good fit models (R-squared 0.602) indicating that there is indeed some relationship between the comments and their frequency and type and benefits. It should be noted though that there is a need for additional evaluation to further support these models. For now, Model 4 can be used cautiously and as a general predictor for benefits due to the small number of cases used in its development. The use of the qualitative level in the models, even as a single predictor, indicates that this is a variable with a strong relationship to the estimated value. It should be pointed out that even though this could be considered as a subjective variable, it was determined based on the nature and number of the comments. In addition, the determination of the quality level requires additional review of the comments either by the reviewer or an independent party and this could be problematic and time consuming. As noted above, and even though the model proposed has a relatively high $\mathrm{R}$-squared value, additional work is needed to ensure the accuracy of the assessment in the future and evaluate Model 4 and possibly include any other predictors. 


\section{CONCLUSIONS}

The work completed here is the first step toward the identification and quantification of the benefits from KYTC constructability reviews. The benefits accrued could be of low monetary amount (most comments resulted in less than $\$ 2,000$ benefit) but there are other intangible benefits such as project delays and scope changes that could not be estimated from the available data. The qualitative analysis of the comments showed that there were few comments with a high severity but those are comments that result in high benefits.

An effort was made to develop prediction models for the benefits accrued based on various attributes of the comments. Even though one could argue that the low number of case studies and comments reviewed may not allow for a robust statistical analysis, the fact that the models developed showed good fit could be considered as a positive indication that the available variables could predict the potential benefits from constructability reviews. Obviously, additional case studies and more comments would enhance these models and possibly allow for a more detailed evaluation of the impact of specific comment types on the review benefits.

The analysis conducted shows a small but significant benefit of 1.25 percent of savings for reviewed projects. Anecdotal evidence indicates that the reviews conducted for KYTC not only provide budgetary benefits but also improve the quality of the constructed projects by defining missing components and correcting errors that could easily lead to increased time and cost for projects. This study indicates that constructability reviews add value to transportation agencies even in streamlined form. Further, there are methods to evaluate and quantify these benefits as well as develop models to predict these benefits. In being able to predict constructability review savings, limited staff can strategically conduct these reviews to optimize their benefit.

\section{REFERENCES}

Anderson, S. and Fisher, D. (1997) Constructability Review Process for Transportation Facilities Workbook, NCHRP Report 390, Transportation Research Board., Washington D.C.

Gambatese, J., Pocock, J., \& Phillip, D. (2007). Constructability: Concepts and Practices. American Society of Civil Engineers, Reston, VA.

Kentucky Transportation Cabinet (KYTC). (2012). Quality Assurance. http://transportation.ky.gov/highway-design/pages/quality-assurance.aspx (12/10/16) Stamatiadis, N., Sturgill, R., Goodrum, P., Shocklee, E., and Wang, C. (2013). Tools for Applying Constructability Concepts to Project Development (Design)-Final Report, Kentucky Transportation Research Center Report KTC-13-14, College of Engineering, University of Kentucky, Lexington, KY. 